\title{
Etude hydrobiologique d'une rivière aménagée : le Verdon Les lacs dle barrage et les tronçons de cours d'eau à débit régullé
}

Hydrobiological study of a harmessed river : the Verdon.

Résumé de l'étude présentée à la Société Hydrotechnique de France par

\section{Alain Grégoire}

Electricité de France

Direction de l'Equipement

Département Sites - Environnement - Information - Paris

(Prix Henri Milon 1982)

La mise en évidence de l'impact de 5 aménagements hydro-électriques sur les composantes d'un écosystème rivière à truite (le Verdon), fait l'objet de 2 grands chapitres consacrés l'un à la situation dans les retenues et l'autre aux problèmes écologiques spécifiques des tronçons de cours d'eau régulés.

Ces deux parties sont précédées d'une description somma ire du bassin versant du Verdon.

\section{Le bassin du Verdon (Fig. 1)}

Le cours du Verdon, long de 155 kilomètres, traverse 3 secteurs géographiques bien marqués, caractérisés par la nature de leur relief et leur distance à la Méditerranée:

- le Haut-Verdon compris entre les sources (3052 m) et la retenue de Castillon $(880 \mathrm{~m})$,
Q Gravière
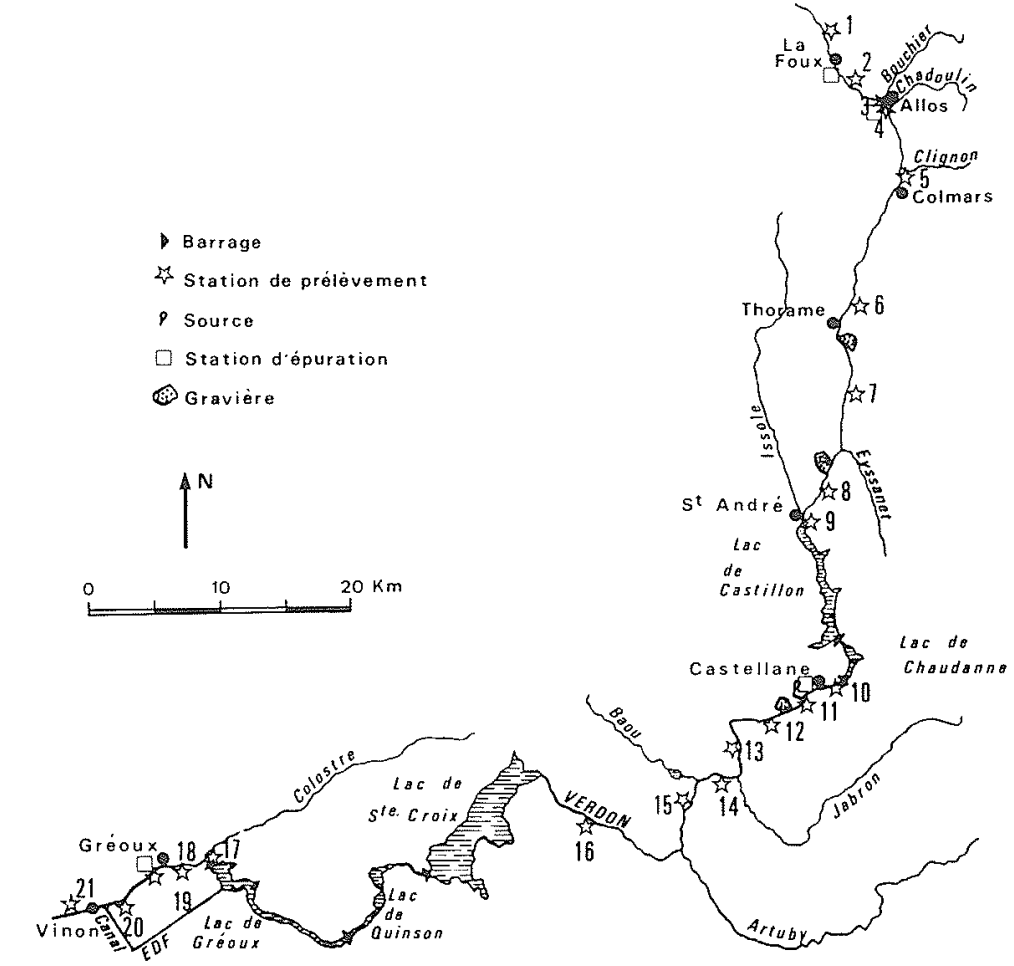

Figure 1 - Le Verdon - Localisation des stations de mesures en eau courante. 
- le Moyen-Verdon coulant au fond des Grandes Gorges entre les retenues de Castillon et de Sainte-Croix ( $480 \mathrm{~m}$ ), - le Bas-Verdon, délimité par la queue de retenue de Sainte-Croix et la confluence Verdon-Durance.

Les terrains de son bassin versant à dominance marnocalcaire renferment des dépôts salifères du Trias.

Les eaux météoriques s'enfoncent profondément dans le substrat très perméable de cette région où le réseau hydrographique de surface est quasi inex istant.

La pente moyenne du Verdon est relativement faible.

De forme très allongée, la vallée présente une succession de zones larges et de gorges correspondant à la nature des terrains traversés.

La proximité de la mer se fait sentir sur tout le cours. Sur la base des valeurs de la pluviosité, la vallée du Verdon est classée dans les climats du type "zone méditerranéenne sublittorale" : été très sec, automne très humide. Le gradient thermique est très marqué, des sources à la confluence avec la Durance.

Le régime pluvio-nival du Verdon présente un débit moyen annuel naturel de $39,5 \mathrm{~m} 3 / \mathrm{s}$ au confluent.

Dans sa partie non aménagée (amont de la retenue de Castillon), les terrains en majorité imperméables rendent le régime tributaire de la pluviosité.

Dans le Grand Canyon, le régime réel du Verdon est très irrégulier car soumis aux lâchures des usines hydroélectriques de Castillon et Chaudanne. Il peut ainsi passer en une journée de $0,5 \mathrm{~m}^{3} / \mathrm{s}$ (débit réservé) à $42 \mathrm{~m}^{3} / \mathrm{s}$ (turbinage maximal). Au cours de l'été 1979 , aucun turbinage n'a été pratiqué à l'usine de Chaudanne et seul le débit réservé a été restitué à la rivière.

L'hydraulicité du Bas-Verdon est aussi sous l'étroite dépendance du programme d'exploitation des barrages EDF. Au droit de la retenue de Gréoux, le Verdon est dérivé de son cours naturel dans un canal dont le débit peut atteindre $55 \mathrm{~m}^{3} / \mathrm{s}$. La plus grande partie, soit $21 \mathrm{~m}^{3} / \mathrm{s}$ en moyenne, est utilisée par la Société du Canal de Provence pour l'alimentation en eau potable et l'irrigation. Le reste retourne à la rivière après turbinage par l'usine hydro-électrique de Vinon, $14 \mathrm{~km}$ plus en aval. Le tronçon court-circuité est alimenté par le Colostre (environ $1 \mathrm{~m}^{3} / \mathrm{s}$ ) et par le débit réservé laissé au barrage de Gréoux ( $1 \mathrm{~m}^{\hat{3}} / \mathrm{s}$ en été, $0,2 \mathrm{~m}^{\overline{3}} / \mathrm{s}$ le reste de l'année).

La nature est la quantité des alluvions transportées par le Verdon diffèrent notablement de l'amont vers l'aval en raison de la diversité des unités géologiques drainées.

Les marnes, formation principale du substrat, très sensibles à l'érosion, sont les constituants dominants des matières en suspension véhiculées par le cours d'eau en période de crues. Les apports en limons dans la retenue de Castillon sont évalués à $40000 \mathrm{~m}^{3} / \mathrm{an}$. Leur décantation dans les retenues explique la faiblesse des apports en matériaux solides à la Durance (quelques milliers de mètres cubes par an).

L'aménagement du bassin versant est peu développé.

Les villages sont peu peuplés et sont pour la plupart équipés de stations d'épuration.

Le tourisme, du fait du sous-développement de son infrastructure, reste limité.

L'agriculture, bien qu'en voie de régression, demeure l'activité principale.

\section{Les retenues hydro-électriques du Verdon}

\section{1. Les composantes de l'environnement}

\section{II.1.1. Composantes abiotiques}

L'examen des données disponibles relatives aux caractéristiques géographiques, géologiques, anthropiques et hydrauliques des 5 aménagements hydro-électriques du Verdon permet de mettre en évidence leur grande diversité.

Les retenues de Castillon, Chaudanne, Sainte-Croix, Quinson et Gréoux, édifiées depuis 1948, sont toutes situées dans le département des Alpes-de-Haute-Provence à des altitudes et des distances à la source du cours d'eau notablement différentes.

Sainte-Croix est la deuxième retenue de France du point de vue de la capacité $\left(767 \mathrm{hm}^{3}\right)$ alors que les bassins de compensation de Chaudanne et Quinson présentent des dimensions beaucoup plus réduites (respectivement $16 \mathrm{hm}^{3}$ et $19,5 \mathrm{hm}^{3}$ ).

Le mode de gestion de ces lacs de barrage est également très divers :

- le marnage peut atteindre $35 \mathrm{~m}$ à Castillon alors qu'il n'est que de $1,5 \mathrm{~m}$ au maximum à Gréoux ;

- le temps moyen de renouvellement est estimé à 280 jours à Sainte-Croix, mais à seulement 7 jours à Quinson.

$\mathrm{La}$ nature géologique à dominance marno-calcaire de leur substrat apparaît comme la seule caractéristique commune.

L'étude des paramètres physiques et chimiques de l'eau et des sédiments des retenues du Verdon a pour objectif principal la mise en évidence des potentialités trophiques du milieu. Dans chaque réservoir, l'emplacement des stations a été choisi après avoir testé le degré d'homogénéité de la masse d'eau. La fréquence des mesures est adaptée aux buts poursuivis et à la variabilité des paramètres considérés.

Par rapport aux lacs naturels, tels ceux d'Auvergne (Lair, 1975), les eaux sont peu transparentes.

Sensiblement identique dans chacune des retenues, le profil thermique permet de les assimiler aux lacs monomictiques chauds de deuxième ordre selon les classifications de Whipple (1927) et de Hutchinson et Loffler (1956). Le budget calorifique est partout impor$\tan t$.

Les eaux sont toujours bien oxygénées. Le profil thermique estival induit une stratification de l'oxygène dissous, mais les teneurs constatées dans l'hypolimnion ne descendent pas au-dessous de $50 \%$ de la saturation.

En été, au voisinage de la surface, l'activité photosynthétique du phytoplancton provoque un accroissement du pH de quelques dizièmes d'unités.

Les eaux sont moyennement minéralisées, bicarbonatées calciques, alcalines et exemptes de pollution organique sensible (Fig. 2). Cependant, dans la retenue de Gréoux et l'anse d'Esparron, la charge en sels nutritifs est accrue du fait d'un déversement direct d'eaux usées.

D'après la classification de Cailleux (1954), les vases sont formées principalement de limons fins et d'argiles. Elles sont, de plus, très riches en sels de calcium mais pauvres en nutrients. 

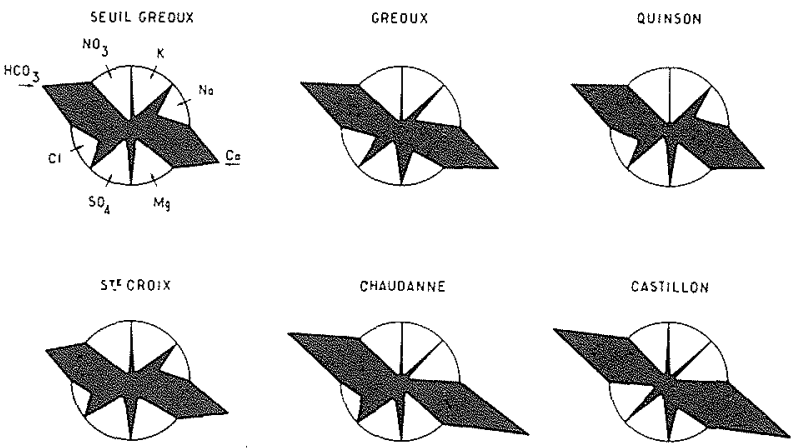

Figure 2 - Composition ionique de l'eau des différentes retenues du Verdon (moyenne annuelle de l'eau de surface).

\section{II.1.2. Composantes biotiques}

Le suivi de l'évolution saisonnière des populations d'organismes correspondant aux principaux maillons de la chaîne alimentaire permet d'évaluer le niveau trophique des différentes retenues du Verdon.

Les populations bactériennes hétérotrophes aérobies strictes ou facultatives sont peu développées et caractéristiques de milieux xénosaprobes à oligosaprobes d'après la classification de Sladecek (1963).

La présence d'Escherichia coli, bactérie indicatrice de pollution fécale, n'est décelée que dans l'anse d'Esparron.

Le degré de trophie d'un lac est également souvent déterminé sur la base de la richesse du peuplement algal évaluée par le biais de la mesure des teneurs en pigments chlorophylliens (Parsons et Strickland, 1963), de la pro. duction primaire au 14 C (Steemann - Nielsen, 1952) ou des densités cellulaires (Utermohl, 1958).

Hormis à Esparron, le développement algal demeure limité, même en période estivale. Selon les classifications en vigueur (Sakamoto, 1966 et Margalef, 1975), les réservoirs étudiés peuvent être considérés en première approximation, comme des plans d'eau oligotrophes. Mis à part Quinson où la productivité reste très pauvre toute l'année, une légère tendance à la mésotrophie se manifeste en été dans ces lacs.

L'examen de la structure des populations phytoplanctoniques confirme ces résultats.

L'étude du périphyton, réalisée à l'aide de substrats artificiels selon la méthode de Clavel et Bouchaud (1980), révèle l'existence d'un peuplement bien diversifié caractérisé par la cohabitation d'espèces phytoplanctoniques et d'espèces périphytiques strictes.

L'absence de macrophytes dans trois des cinq retenues et leur limitation à quelques secteurs des lacs de Quinson et Gréoux sont parmi les particularités les plus remarquables de ces milieux artificiels.

Cinq espèces cosmopolites ( 3 Cladocères et 2 Copépodes) dominent le zooplancton récolté dans les lacs de barrage du Verdon. Cependant, seuls les Cladocères sont représentés à Castillon.

La densité des communautés demeure généralement limitée. Les plus fortes valeurs sont obtenues à SainteCroix avec 1000 ind. $/ \mathrm{m}^{3}$.

Les zooplanctontes colonisent toute la colonne d'eau mais les effectifs les plus abondants, lors de nos prélèvements effectués en milieu de journée, sont recensés aux alentours de 5 mètres de profondeur.
Le peuplement semble plus important l'hiver dans Sainte-Croix et Gréoux. C'est le phénomène inverse qui apparaît à Castillon.

Comme dans la très grande majorité des réservoirs et notamment ceux de France tels que Cajarc (Gagneur, 1976), Serre-Ponçon (Nino, 1979), Chastang (Lair et al., 1980), la faune benthique comprend essentiellement des larves de Chironomides et des Oligochètes répartis presque exclusivement dans les sédiments argilolimoneux (Fig. 3). Le zoobenthos a été recensé à toutes les profondeurs mais nos observations montrent que la contribution relative des larves de Diptères à la densité totale est fonction de la hauteur d'eau au-dessus du fond et de la granulométrie du substrat. Ainsi peut s'expliquer la dominance des Chironomides dans les sédiments relativement grossiers des retenues de Gréoux et Quinson; à Castillon, Chaudanne et Sainte-Croix, les Oligochètes sont par contre favorisés par les vases à particules fines. Ce mode de répartition a été remarqué par ailleurs aux Etats-Unis par Thut (1969), Ryals (1975), Edmonds et Ward (1979).
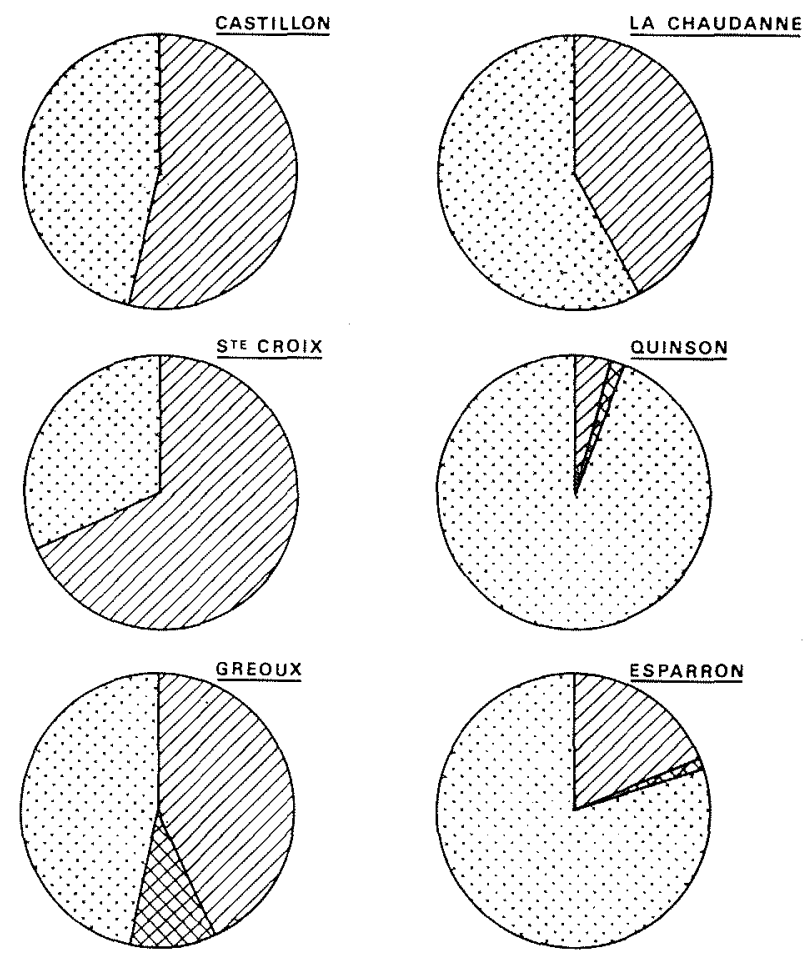

$\because$ Chironomides

Q77 oligochètes

$x \times$ Autres

Figure 3 - Importance numérique relative des différents groupes d'Invertébrés benthiques dans les retenues du Verdon.

Le peuplement benthique est peu dense à Castillon et à Chaudanne (moins de 1000 ind.$/ \mathrm{m}^{2}$ ). Dans les 3 autres lacs, les effectifs atteignent de l'ordre de 3000 ind. $/ \mathrm{m}^{2}$.

L'inventaire de la faune ichtyologique de ces lacs a été dressé en 1976 et en 1977 et la structure du peuplement piscicole étudiée (Champeau, Grégoire et Brun, 1979). Les engins de pêche utilisés ont été principalement des filets.

Dix-neuf espèces de poissons ont été recensées (Fig. 4). Les Cyprinidés sont dominants (toxostomes, 

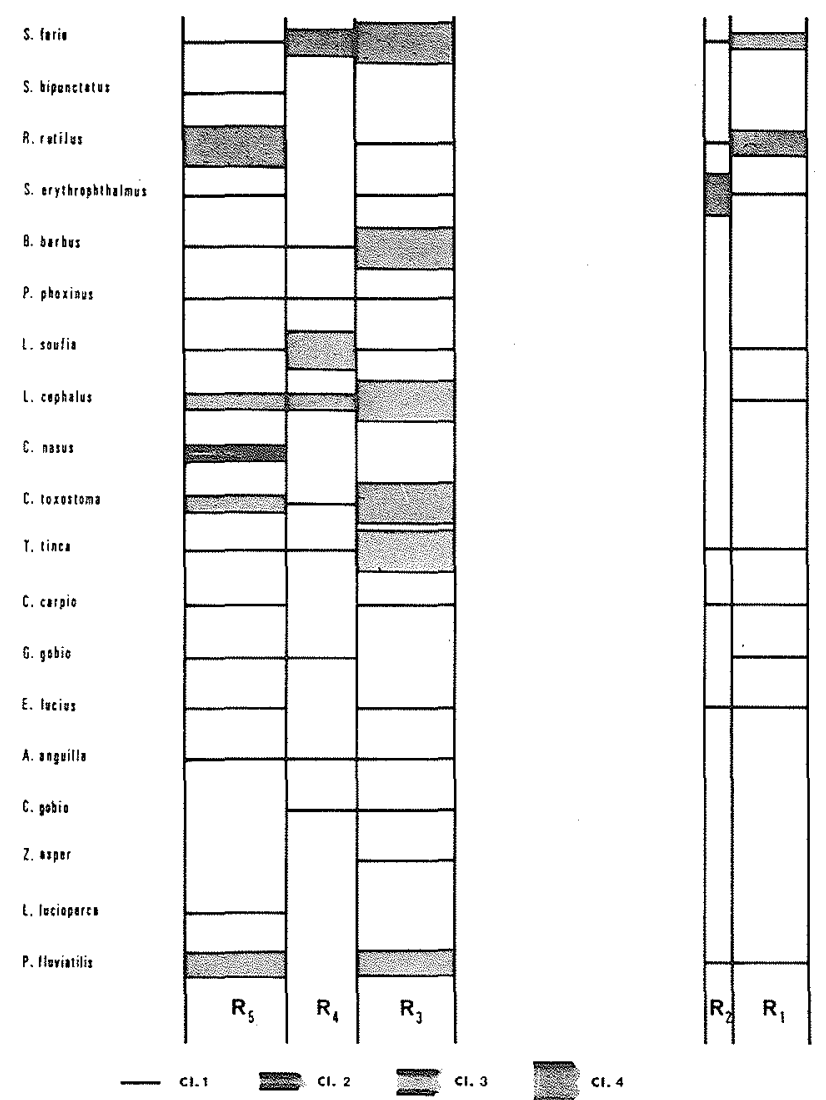

Figure 4 - Répartition quantitative des poissons des retenues du Verdon. Cl. 1 à $\mathrm{Cl} .4$ : classes d'abondance croissantes; R1= retenue de Castillon, $R 2=$ retenue de Chaudanne, $R 3=$ retenue de Sainte-Croix, $R 4=$ retenue de Quinson, $R 5=$ retenue de Gréoux.

chevesnes, gardons), mais à Sainte-Croix et Quinson, les populations de truites sont aussi très importantes. Quatre groupements d'espèces, en relation étroite avec le schéma d'aménagement hydro-électrique du Verdon, ont été mis en évidence. Le calcul de la diversité spécifique et de l'équitabilité, pour chacune des retenues, montre que deux d'entre elles possèdent une population de poissons relativement équilibrée alors que dans les trois autres une espèce domine.

L'examen de la fréquence des tailles et de la relation longueur-poids, pour les espèces dominantes, montre qu'en se référant aux données de Spillman (1961) et Laurent et Moreau (1973), la croissance est plus importante dans les retenues aval (Sainte-Croix, Quinson et Gréoux). Dans le lac de Sainte-Croix, ces calculs confirment l'existence d'une forte corpulence chez les truites.

\section{II.2. Les grands problèmes d'ordre écologique spéci- fiques des lacs de barrage}

L'étude synécologique des lacs de barrage du Verdon montre qu'en plus d'un temps de renouvellement très court, l'originalité de ces milieux par rapport aux lacs naturels, résulte principalement de l'existence de 3 phénomènes :

- modalités de formation d'un écosystème lacustre après la mise en eau du réservoir,
- influence du marnage sur les différentes composantes de cet écosystème,

- impact des vidanges décennales sur ces mêmes facteurs.

\section{II.2.1. Evolution de l'écosystème lacustre dans les pre- mières années suivant la mise en eau}

Lors de l'inondation de la cuvette de la future retenue, la végétation laissée en place se décompose en consommant de l'oxygène et en libérant des sels nutritifs. La stimulation de la productivité des divers niveaux de la pyramide trophique qui en résulte, dure environ 2 ans. Puis une phase dépressive, attribuée à l'épuisement de la "nourriture" (Baranov, 1961), précède souvent l'installation d'un équilibre relatif. Ce processus classiquement rapporté, de Lokhanina (1959) pour les planctontes à Cherry et Guthrie (1975) pour les poissons, a été particulièrement perceptible lors des mises en eau de Ste-Croix et Quinson, sur le plancton et l'ichtyofaune.

\section{II.2.2. Impact du mamage sur l'écosystème lacustre}

Contrairement à un lac naturel, les retenues sont soumises à des fluctuations de niveau souvent très importantes (jusqu'à 35 mètres à Castillon). Leur influence sur les biocénoses aquatiques résulte de l'accroissement de la turbidité des eaux et surtout de l'exondation de grandes superficies de la cuvette du lac (tableau I). Comme dans la plupart des retenues françaises étudiées, les organismes les plus perturbés sont les algues dont l'efficience photosynthétique est réduite ainsi que les macrophytes. En outre, certains groupes d'Invertébrés benthiques (Odonates, Ephéméroptères, Lamellibranches, Amphipodes) sont totalement éliminés et comme le rapportent de nombreux auteurs comme Hynes (1961), Marshall (1978), Kaster et Jacobi (1978), seules quelques populations peu diversifiées d'Oligochètes et de larves de Chironomides sont capables de résister à ces conditions rigoureuses. Ces organismes peuvent en effet survivre à un marnage prolongé d'hiver grâce à leurs facultés de résistance à la dessiccation (Nino, 1979 -Lamberti et Nino, 1979- Champeau et al., 1980).

Tableau 1. - Superficie des zones découvertes par les baisses de niveau des retenues

\begin{tabular}{|l|c|c|c|}
\hline Paramètres & $\begin{array}{c}\text { Surface totale } \\
\text { S.T. (ha) }\end{array}$ & $\begin{array}{c}\text { Surface maximale } \\
\text { découverte } \\
\text { S.D. (ha) }\end{array}$ & $\begin{array}{c}\text { S.T./S.D. } \\
\%\end{array}$ \\
\hline Castillon & 200 & 110 & 55 \\
Chaudanne & 65 & 25 & 38 \\
Sainte-Croix & 2182 & 600 & 27 \\
\hline
\end{tabular}

L'effet de la diminution des effectifs des populations d'Invertébrés en tant que stock de nourriture potentielle pour les poissons a été analysé par Benson (1973b) dans des réservoirs du Missouri.

Pour les poissons, l'impact du marnage peut être direct par assèchement des oufs lors des baisses de niveau de l'eau. Vivier et al. (1956) à Eguzon, Lowe - Mc Connell (1973) dans une étude synthétique sur le sujet ont également posé le problème. 


\section{II.2.3. Impact des vidanges décennales sur l'écosystème lacustre}

Les observations réalisées lors de la vidange décennale de Chaudanne (Grégoire, 1975) permettent de montrer l'impact de cette opération sur la qualité de l'eau du lac, d'une part et sur le tronçon de rivière aval, d'autre part. Dans les réservoirs, les Invertébrés benthiques ne sont pas perturbés; les organismes pélagiques (planctontes et poissons) sont, par contre, totalement éliminés.

\section{II.3. Place des retenues du Verdon dans le parc hydrau- lique français}

En vue d'une éventuelle prévision de l'évolution de la qualité des eaux d'autres retenues, le degré de signification des résultats est évalué par la recherche de la place des cinq lacs du Verdon dans le parc hydraulique français. Les données d'ordre biotiques relatives aux lacs de barrage étant trop peu nombreuses, cette classification est effectuée à partir de leurs caractéristiques physiques.

Le choix des 22 descripteurs abiotiques destinés à expliquer la structure biologique des écosystèmes lacustres est basé sur les travaux de Thienemann, 1925 Naumann, 1932 - Vivier et al., 1956 - Dussart, 1966 - Feuillade et al., 1971 - Benson, 1973a - Macan, 1974 - Walker et Lawson, 1977 - Loucks et Odum, 1978 - Lund, 1978 - Straskraba, 1978 - Uzabiaga, 1978.

Une analyse des corrélations entre paramètres montre que ces descripteurs peuvent être partitionnés en 5 groupes représentant respectivement les apports à la retenue, la morphométrie du réservoir, le mode d'exploitation de l'usine, les spécifications techniques des installations et les données qualitatives diverses.

La classification automatique de Diday (1970) définit 7 types de retenues (tableau II) dont la différenciation est expliquée par l'analyse factorielle des correspondances multiples mise au point par Benzécri (1972).

Les aménagements du type 1 dont l'élément le plus représentatif est Tuillière sur la Dordogne, ont une puissance maximale possible assez faible (de 5 à $50 \mathrm{MW}$ ). Les retenues, toutes situées à basse altitude, à une distance à la source du cours d'eau supérieure à $300 \mathrm{~km}$, sont alimentées par un bassin versant important (4000 à $20000 \mathrm{~km}^{2}$ ). Le débit moyen annuel des apports est relativement élevé $\left(200\right.$ à $\left.600 \mathrm{~m}^{3} / \mathrm{s}\right)$; il est la principale cause du renouvellement rapide du plan d'eau (inférieur à 24 heures) et de l'absence d'un marnage notable.

Les usines "au fil de l'eau" du Rhin et du Rhône sont regroupées dans le type 2 . Construites en plaine, sur le cours inférieur de fleuves à fort débit (supérieur à 600 $\mathrm{m}^{3} / \mathrm{s}$ ), leur productibilité en année moyenne est supérieure à $500 \mathrm{GWh}$. Le mode de fonctionnement de ces usines implique un temps de renouvellement de la retenue inférieur à la journée et des variations de niveau du plan d'eau négligeables. Les ouvrages de ce type sont dits "à buts multiples" puisqu'ils servent aussi à l'amélioration des conditions de navigation par régularisation du régime pluvio-nivo-glaciaire du fleuve.

Le type 3 est formé par des retenues situées en haute altitude, sur le cours supérieur des rivières. En relation avec la très faible étendue du bassin versant (moins de $100 \mathrm{~km}^{2}$ ), les apports en eau sont faibles (inférieurs à $5 \mathrm{~m}^{3} / \mathrm{s}$ et irréguliers (régime glaciaire à nival). Les aménagements de l'Ariège et des Hautes-Pyrénées tels Izourt, Pla de Gnioure, les Gloriettes, Araing, Migoelou. . . sont caractéristiques de ce type.

Dans le type 4 sont regroupées les plus grandes retenues de France, tant du point de vue profondeur (de l'ordre de la centaine de mètres) que superficie (de l'ordre du millier d'hectares) ou capacité (supérieure à $200 \mathrm{hm}^{3}$ ). On retrouve ainsi dans ce groupe, outre Monteynard le réservoir le plus représentatif du type, les lacs de barrage de Ste-Croix, de Castillon et de SerrePonçon. La puissance installée et la puissance maximale possible sont, pour ces aménagements, très fortes puisque respectivement supérieures à $200 \mathrm{MVA}$ et $200 \mathrm{MW}$.

Les retenues du type 5 sont surtout localisées dans le Massif Central : Saints-Peyres, Pont-de-Salars, Maury, Marcillac-Brigoux, la Triouzoune... Elles sont placées sur le tronçon amont des cours d'eau, à moyenne altitude $(600$ à $1000 \mathrm{~m})$. Les débits d'alimentation du lac de barrage (inférieurs à $5 \mathrm{~m}^{3} / \mathrm{s}$ en moyenne annuelle) sont en relation avec la faible superficie du bassin versant $\left(100\right.$ à $\left.1000 \mathrm{~km}^{2}\right)$. Les usines de ce type ont un débit maximal turbinable et une productibilité faibles, respectivement 5 à $35 \mathrm{~m}^{3} / \mathrm{s}$ et 10 à $100 \mathrm{GWh}$. Les retenues correspondantes, de capacité et de profondeur moyennes (respectivement 11 à $50 \mathrm{hm}^{3}$ et 30 à $90 \mathrm{~m}$ ), se renouvellent entre 1 et 3 mois et sont soumises à un marnage supérieur à $20 \mathrm{~m}$.

Les retenues constitutives du type 6 sont plus éloignées de la source que les précédentes (entre 50 et $150 \mathrm{~km}$ ). La superficie de leur bassin versant (de l'ordre de $1000 \mathrm{~km}^{2}$ ) ainsi que le débit des rivières qui les alimentent (entre 5 et $35 \mathrm{~m}^{3} / \mathrm{s}$ ) sont ainsi normalement plus importants. Les débits maximaux turbinables sont compris entre 5 et $35 \mathrm{~m}^{3} / \mathrm{s}$ pour une productibilité en année moyenne de 10 à $100 \mathrm{GWh}$. Les conditions d'exploitation des usines hydro-électriques font que les variations de niveau sont en général importantes (de 5 à $20 \mathrm{~m}$ ) et le temps de renouvellement faible ( 25 à 600 heures). Les retenues de Chaudanne, Quinson, Gréoux et Chastang appartiennent à ce type.

Bien que situés à faible altitude $(200-600 \mathrm{~m})$, les réservoirs du type 7 ont été construits sur le tronçon amont des cours d'eau. Draînant un bassin versant de faible étendue $\left(100\right.$ à $\left.1000 \mathrm{~km}^{2}\right)$, le débit moyen annuel des apports est faible puisqu'inférieur à $5 \mathrm{~m}^{3} / \mathrm{s}$, ma is régulier du fait de l'influence du climat océanique. Les retenues, du fait de leurs tailles très modestes (capacité inférieure à $10 \mathrm{hm}^{3}$ ), renouvellent leurs masses d'eau en moins d'un mois.

Les lacs du Verdon se répartissent dans les types 4 et 6 qui représentent $42 \%$ de l'ensemble du parc.

\section{Impact des aménagements hydro-électriques sur l'écosystème rivière}

Les trois tronçons d'eau courante du Verdon, individualisés par l'édification des barrages hydro-électriques, sont caractérisés par des régimes hydrauliques très différents : 
Tableau II. - Typologie des retenues françaises - composition des types

TYPE $1-22$ retenues, soit $11,9 \%$ de l'ensemble étudié

TUILLIERE (Dordogne); SEYSSEL (Ain); CUSSET-JONS (Ain); LUZECH (Lot); VILLENEUVE-SUR-LOT (Lot-et-Garonne); TEMPLE (Lot-et-Garonne); LA VANELLE (Drôme); CHAUTAGNE (Haute Savoie); BELLEY (Ain); BREGNIER-CORDON (Ain); LOYETTES (Ain); PIZANCON (Drôme); SAINT-HILAIRE (Isère); SAINT-JULIEN (Haute-Garonne) ; BEAUVOIR (Isère); SAULTBRENAZ (Ain); CADARACHE (Bouches-du-Rhône); ESCALE (Alpes-de-Haute-Provence); SALIGNAC (Alpes-de-Haute-Provence); MANCIOUX (Haute-Garonne) ; GENISSIAT (Haute-Savoie); MALAUSE (Tarn-t-Garonne).

TYPE 2 - 10 retenues, soit $5,4 \%$ de l'ensemble étudié.

MARCKOLSHEIM (Bas-Rhin); RHINAU (Bas-Rhin); PIERRE BENITE (Rhône); GERSTHEIM (Bas-Rhin)); KEMBS (Haut-Rhin); LE PEAGE-DE-ROUSSILLON (Isère); STRASBOURG (Bas-Rhin); VAUGRIS (Isère); BOURG-LES-VALENCE (Drôme); VALLABREGUES (Gard).

TYPE 3 - 23 retenues, soit $12,4 \%$ de l'ensemble étudié.

IZOURT (Ariège); PLA DE GNIOURE (Ariège); LES GLORIETTES (Hautes-Pyrénées); ARAING (Ariège); MIGOELOU (HautesPyrénées); GREZIOLLES (Hautes-Pyrénées); NAGUILlES (Ariège); LA GIROTTE (Savoie); PORTILLON (Haute-Garonne); AUBERT (Hautes-Pyrénées); BISSORTE (Savoie) ; SAUT-DE-VESOLES (Hérault); CAP-DE-LONG (Hautes-Py rénées); ESCOUBOUS (Hautes-Pyrénées); PLAN-D'AVAL (Savoie); PLAN-D'AMONT (Savoie). SASSIERE (Savoie); BOUVANTE (Drôme); LA FOUS (Alpes-Maritimes); GRANDES PATURES (Ariège); LANOUX (Pyrénées Orientales); LAC LONG (Alpes-Maritimes); SAINT-MARTIN VESUBIE (Alpes-Maritimes).

TYPE 4 - 19 retenues, soit 10,3\% de l'ensemble considéré.

MONTEYNARD (Isère) ; SARRANS (Aveyron) ; GRANDVAL (Cantal) ; L'AIGLE (Cantal) ; LE MOTTY (Hautes-Alpes) ; BORT-LESORGUES (Corrèze); VOUGLANS (Jura); TIGNES (Savoie); MONT-CENIS (Savoie) ; CASTILLON (Alpes-de-Haute-Provence) ; LA BATHIE (Savoie) ; SERRE-PONCON (Hautes-Alpes) - MOUSTIERS (Alpes-de-Haute-Provence) ; RABUONS (Alpes-Maritimes) ; SAINTGUERIN (Savoie); GRAND'MAISON (Isère) ; SAINTE-CROIX (Alpes-de-Haute-Provence); VILLEREST (Loire); LA GITTAZ (Savoie).

TYPE 5 - 31 retenues, soit 16,8\% de l'ensemble étudié.

SAINTS-PEYRES (Tarn); PONT-DE-SALARS (Aveyron); MAURY (Aveyron); MARCILLAC-BRIGOUX (Corrèze); LA TRIOUZOUNE (Corrèze); MONCEAUX-LA-VIROLE (Corrèze); LAOUZAS (Tarn); VILLEFORT (Lozère); ROUJANEL (Lozère); BAGE (Aveyron); ENCHANET (Cantal); PUYVALADOR (Pyrénées-Orientales); LA RAVIEGE (Tarn); GUERLEDAN (Côtes-du-Nord); RASCHAS (Lozère); VILLEFRANCHE-DE-PANAT (Aveyron); LA PALISSE (Ardèche); LE GAGE (Ardèche) ; SAINT-ETIENNECANTALES (Cantal); LAVAUD-GELADE (Creuse); LE SAUTET (Isère); LASTIOULLES (Cantal); SAINT-CASSIEN (VaT) ; LE CHAMBON (Isère); LE VERNEY (Isère); PARELOUP (Aveyron); VASSIVIERES (Haute-Vienne); TOLLA (Corse du Sud); MATEMALE (Pyrénées-Orientales); CALACUCCIA (Haute-Corse); LAC NOIR (Haut-Rhin).

TYPE 6 - 58 retenues, soit $31,4 \%$ de l'ensemble étudié.

LE GOUR NOIR (Corrèze); QUEUILLE (Puy-de-Dôme); LES CHOMETTES (Corrèze); LA ROCHE-TALAMIE (Creuse) ; SAINTMARC (Haute-Vienne); L'ETROIT (Creuse); HAUTEFAGE (Corrèze); MONTLARRON (Haute-Vienne); ROCHEBUT (Allier); CASTELNAU-LASSOUTS (Aveyron); MOUX (Ain); TREIGNAC (Corrèze); POUTES (Haute-Loire); CAMBEYRAC (Aveyron); VALLIERES (Haute-Savoie); NEPES (Cantal); PINET (Aveyron); VAUSSAIRE (Cantal); LA CHAUDANNE (Alpes-de-HauteProvence); THURIES (Tarn); SAINT-PIERRE-COGNET (Isère); CIZE-BOLOZON (Ain); CHATEL-MONTAGNE (Allier); VEZINS (Manche); ALLEMENT (Ain); RABODANGES (Orne); LANAU (Cantal); NOTRE-DAME-DE-COMMIERS (Isère); COUESQUE (Aveyron); SAUT-MORTIER (Jura); VAUFREY (Doubs); MOTZ-VAL-DE-FIER (Savoie); EGUZON (Indre); MALARCE (Ardèche); LE PRAT (Allier); LE TRUEL (Aveyron); LA JOURDANIE (Aveyron); BRUGALE (Lot); LABARRE (Ariège); LA ROCHE-AUMOINE (Indre); BIOGE (Haute-Savoie); ARGENTAT (Corrèze); QUINSON (Alpes-de-Haute-Provence); GRANGENT (Loire); COISELET (Ain); RIVIERES (Tarn); GOLINHAC (Aveyron); ESPINASSES (Hautes Alpes); GOUL (Aveyron); EMBRUN (HautesAlpes); CANDES (Lot); GREOUX (Alpes-de-Haute-Provence); LA BARTHE (Aveyron); LE CHASTANG (Corrèze); LA SAUSSAZ (Savoie) ; FLUMET (Isère) ; LE REFRAIN (Doubs) ; LA COCHE (Savoie).

TYPE $7-22$ retenues, soit $11,9 \%$ de l'ensemble étudié

CONFOLENT-LES-COMBES (Creuse); MARTINEIX (Haute-Vienne); BÜJALEÜÜ (Haute-Vienne) ; VILLLJOUBERT (Haute-Vienne) ; PONT-ROLLAND (Côtes-du-Nord); LARTIGE (Haute-Vienne); CRESCENT (Yonne); ROPHEMEL (Côtes-du-Nord); FAUX-LAMONTAGNE (Creuse); LA ROCHE-QUI-BOIT (Manche); CHAUMECON (Nièvre); LA BOURBOULE (Puy-de-Dôme); ESTAENS (Espagne); CHAMMET (Creuse); BIMONT (Bouches-du-Rhône); LE TECH (Hautes-Pyrénées); GROSBOIS (Doubs);VIEUX-PRE (Meurthe-et-Moselle); LES MESCES (Alpes-Maritimes); REVIN-LES-MARQUISADES (Ardennes); VALLON DOL (Bouches-duRhône), FORT-LA-SAULCE (Hautes-Alpes).

- naturels dans le Haut-Verdon,

- influencés par le mode de gestion des ouvrages hydroélectriques dans les Moyen et Bas-Verdon.

L'impact des barrages sur l'écosystème rivière est recherché par la prise en compte de 16 composantes abiotiques et de 6 grands groupes d'indicateurs biologiques.

Les réductions de débit entrainent des modifications parfois importantes des facteurs morphodynamiques du cours d'eau puisque les sections d'écoulement et les vitesses de courant peuvent diminuer de plus de la moitié.

\section{1. Impact sur les composantes abiotiques}

La composition physico-chimique de l'eau de la rivière est fortement influencée par la nature géologique de son bassin versant. Les terrains marno-calcaires expliquent les fortes teneurs en carbonate de calcium.

Les retenues jouent un rôle tampon important. 
Comme l'ont rapporté de nombreux auteurs dont Petr (1978) sur le Nil, Hoden (1979) sur le Colorado, ainsi que Webster, Benfield et Cairns (1979) sur la New River, les retenues font souvent fonction de bassin de décantation pour les matières en suspension véhiculées par le cours d'eau ; ce processus est particulièrement net sur le Haut-Verdon, au droit de la retenue de Castillon. Les concentrations de l'eau en ions chlorures et sulfates présentent également des teneurs plus faibles après un séjour de l'eau dans les réservoirs de Sainte-Croix, Quinson et Gréoux.

Si la capacité de dilution est augmentée dans les retenues, elle est par contre généralement diminuée à l'aval des barrages du fait de la réduction du débit.

La pollution organique décelée dans le Bas-Verdon a pour origine l'assainissement insuffisant des villages riverains auquel s'ajoute l'activité agricole du bassin versant du Colostre (Grégoire, Rivier, Rondon, 1975). La faiblesse des débits restitués au barrage de Gréoux accentue cette tendance. Toutefois, du fait de la bonne oxygénation de la couche hypolimnique, l'altération de la qualité de l'eau, par diminution du pouvoir diluant des rejets domestiques et agricoles, se trouve limitée à l'aval des barrages de Chaudanne et Gréoux malgré la restitution de débits réservés très faibles. La stratification thermique de la masse d'eau et la situation des prises d'eau au voisinage du fond des lacs sont la cause du maintien des basses températures de l'eau de restitution. De nombreux auteurs dont Ward (1976b), Gore (1977), Holden (1979) ont observé de tels phénomènes sur des rivières régulées aux Etats-Unis.

\section{III.2. Impact sur les composantes biotiques}

Les organismes aquatiques choisis comme indicateurs biologiques de l'impact des barrages sur le Verdon sont les Diatomées, les Ephéméroptères et les Amphipodes, groupes les mieux représentés sur l'ensemble du cours d'eau. Ils occupent des niches très variées et présentent, suivant les espèces, une sensibilité différente aux conditions du milieu (Verneaux, 1973 - Coste, 1974 - Ward et Short, 1978). Fixés ou peu mobiles, ils subissent en outre, en permanence, l'influence des barrages.

L'évolution de la structure des populations de Diatomées est suivie le long du Verdon, à l'aide de substrats artificiels immergés, en tenant compte de l'emplacement des apports et des barrages.

L'impact des aménagements hydro-électriques se présente sous deux formes liées à leur type d'exploitation. Le régime d'éclusées entraine une réduction de la biomasse des Diatomées mais un maintien de la diversité spécifique, alors que la restitution d'un débit réservé faible et constant permet le développement d'une flore algale relativement abondante mais peu diversifiée. Dans ces deux cas de figures, les effets induits par les barrages sont amortis vers l'aval par les autres composantes du milieu (Millerioux, Grégoire et Champeau, 1981).

Le calcul de la diversité spécifique des larves d'Ephémères dans chacune des 21 stations de prélèvements montre que des variations journalières brusques de débit $\left(0,5\right.$ à $\left.40 \mathrm{~m}^{3} / \mathrm{s}\right)$ ne modifient pas de manière significative la diversité spécifique. Ward (1976b) a constaté le même phénomène aux Etats-Unis. Comme le rappor- tent Spence et Hynes (1971), Lemkuhl (1972), Ward (1976a), dans le cas d'un débit réservé faible et régulier, la diversité spécifique chute.

Les densités de larves d'Ephémères à l'aval des barrages du Verdon ont toujours été plus faibles qu'à l'amont. Certains auteurs (Minshall et Winger, 1968 . Gore, 1977) attribuent ce fait à l'accroissement du drift, d'autres comme Lemkuhl (1972), Ward (1974, 1976b), Gore (1977, 1980) par exemple, corrèlent les faibles densités d'organismes et les basses températures.

La détermination * de la composition spécifique des populations dans les stations situées de part et d'autre des ouvrages hydro-électriques, permet de constater l'existence, à l'aval immédiat des barrages, de communautés particulières inféodées à un régime hydraulique donné : dominance d'Heptageniidae dans le cas d'un régime d'éclusées, de Baetidae dans le cas de faibles débits constants (Radford et Hartland - Rowe, 1971 - Fisher et Lavoy, 1972 - Grégoire et Champeau, 1981).

Un essai de regroupement des stations de prélèvements sur la base de leur peuplement en Ephémères, met en évidence une partition définie par l'implantation des aménagements hydrauliques. La connaissance des principaux facteurs mésologiques permet d'expliquer la composition des différents groupes obtenus. On constate ainsi la dominance des espèces polluo-résistantes à l'aval des rejets d'origine urbaine ou agricole lorsque le débit réservé de la rivière est très faible.

Les Crustacés (Amphipodes et Entomostracés) sont particulièrement abondants à l'aval des barrages du Verdon quelles que soient les modalités de gestion des usines. Ward et Short (1978) ainsi que Ward et Stanford (1979) ont observé un phénomène similaire dans le Colorado.

Les larves d'Odonates et les vers Hirudinés sont par contre éliminés des tronçons de cours d'eau soumis au régime d'éclusées.

Bien que les mesures des facteurs morphodynamiques et hydrauliques des Gorges du Verdon montrent qu'aucune variation notable des superficies de frayères potentielles, suite à des modifications du débit ne soit à craindre, les effectifs de truites sont environ $80 \%$ moins élevés dans les tronçons régulés. Plusieurs causes ont été invoquées pour expliquer cette situation:

- obstacle à la migration des poissons matérialisé par le barrage,

- altération de la qualité de l'eau,

- perturbation des frayères.

\section{III.3. Circonstance exceptionnelle - Vidange totale de la retenue de Chaudanne (Fig. 5).}

L'influence, sur les composantes abiotiques et biotiques du cours d'eau, de la vidange décennale totale d'une retenue (Chaudanne), est également évaluée. A l'image des modifications survenues en 1956 dans le Rhône lors de la vidange de la retenue de Verbois (Nisbet, 1961), cette situation exceptionnelle s'est surtout traduite par l'apparition de très fortes concentrations en matières en suspension (jusqu'à $50 \mathrm{~g} / \mathrm{l}$ ) et une désoxygénation totale de l'eau immédiatement sous le barrage. Puis la pollution a diminué graduellement vers l'aval (Grégoire, 1975). 


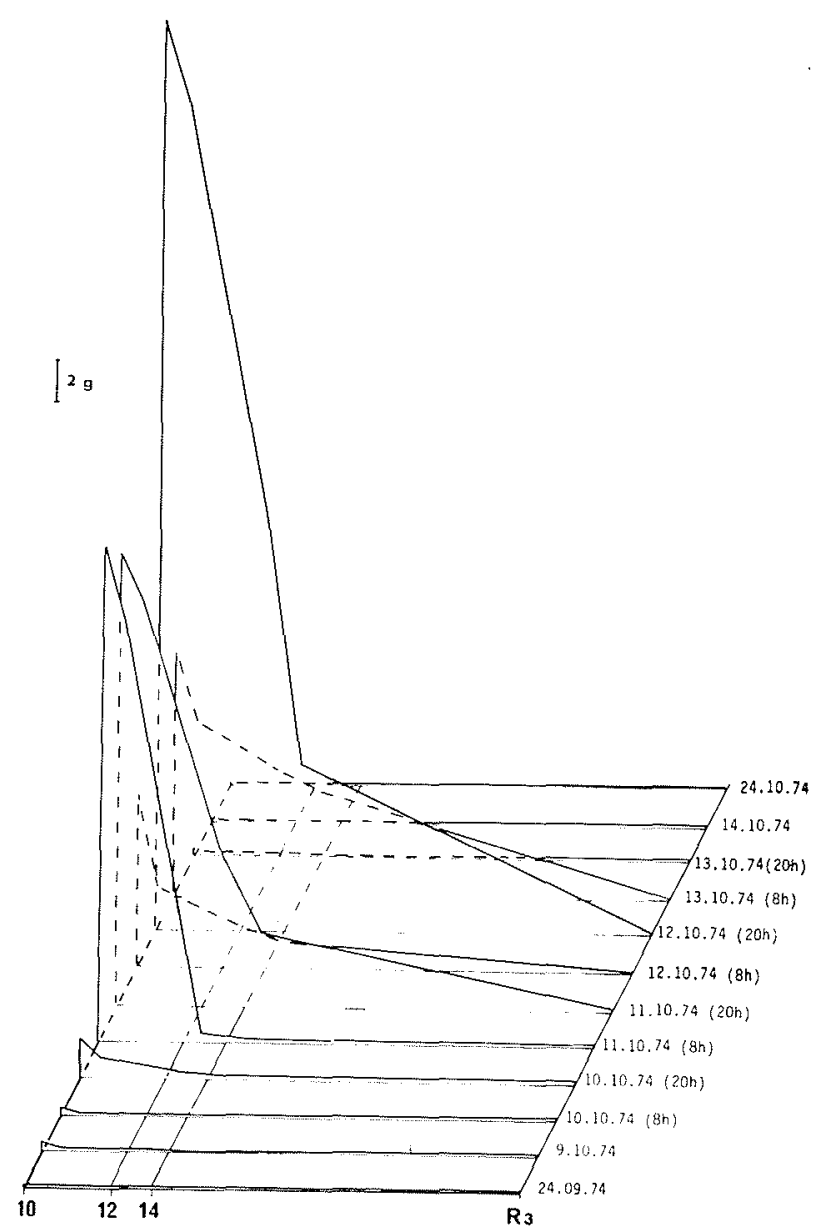

Figure 5 - Variation des teneurs en matières en suspension de l'eau dans les stations du Moyen-Verdon pendant la vidange de Chaudanne. st. 10 : Castellane ; 12 : Chasteuil ; 14 : Point Sublime; R3 : Ste-Croix.
L'impact sur la faune apparaît de façon spectaculaire au niveau des poissons qui ont péri en grand nombre dans la zone la plus polluée. Les mesures ont montré que l'onde de pollution n'a pas dépassé la queue de la retenue de Sainte-Croix.

L'influence à long terme de cette vidange sur le Verdon est très minime. Les analyses chimiques et biologiques effectuées un mois après ne décèlent pratiquement aucune modification de la qualité physicochimique et biologique de l'eau par rapport à l'état initial. Seule la station la plus amont n'a été recolonisée que 8 mois plus tard.

\section{III.4. Analyse mathématique (Fig. 6)}

L'impact respectif des différents barrages sur l'ensemble des composantes de l'écosystème eau courante du Verdon est recherché par le biais de la comparaison des structures mésologiques et biologique des stations de mesures.

Le traitement mathématique utilisé, "l'analyse factorielle des correspondances multiples", met en évidence les affinités réciproques des espèces et des stations et permet la détermination et la quantification des paramètres explicatifs de ces ressemblances.

Tous les paramètres sont traités en variables actives. La distribution des éventualités significatives et des stations dans les plans principaux d'inertie met nettement en évidence :

- la présence, à l'aval immédiat des barrages, d'espèces planctoniques originaires du plan d'eau amont,

- la sélection, dans les tronçons régulés, d'espèces d'organismes aquatiques en fonction du mode de gestion des ouvrages hydro-électriques.

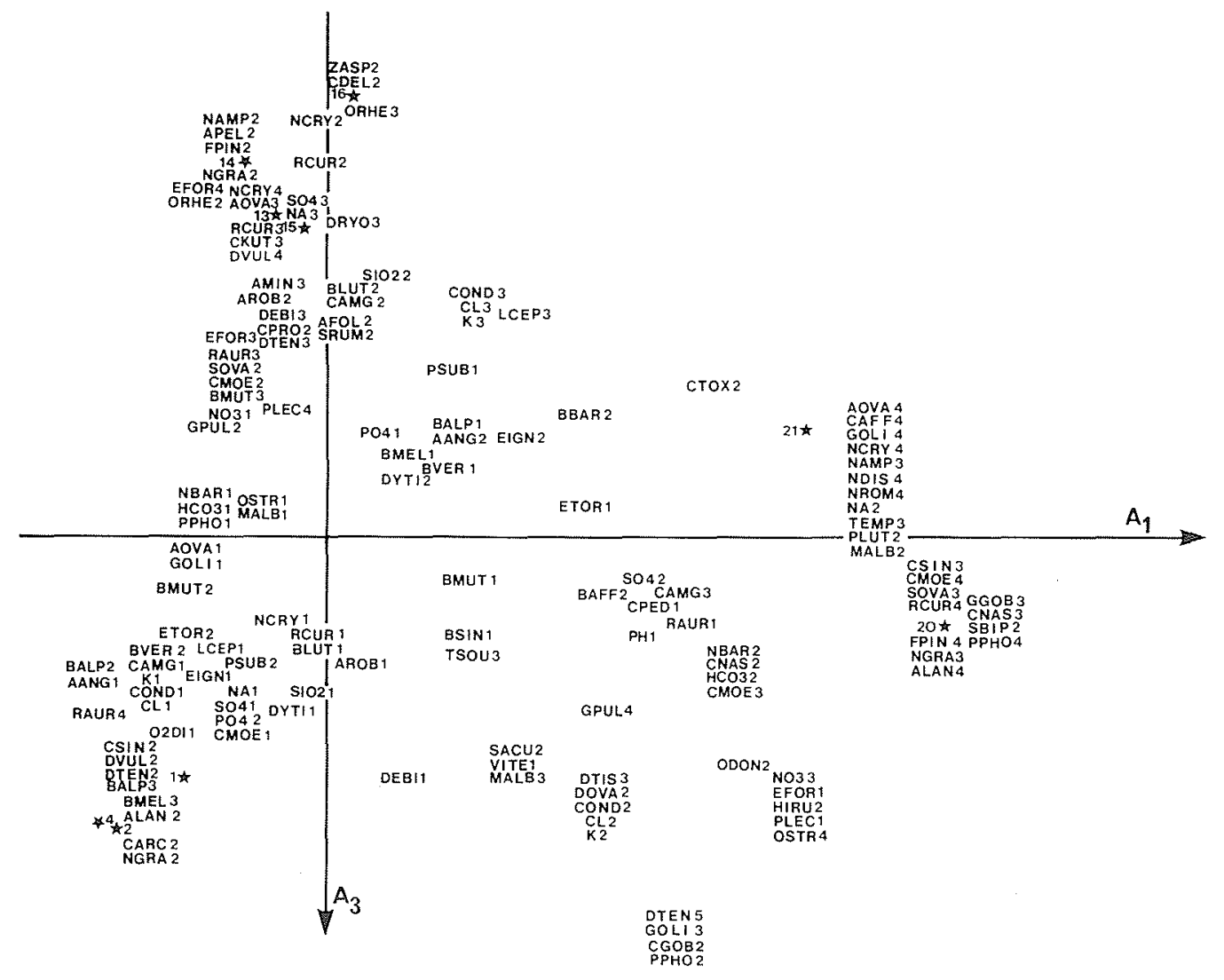




\section{Conclusion - Considérations relatives à la nécessité d'un choix de l'objectif de qualité d'un cours d'eau}

Les principales règles de gestion des aménagements hydro-électriques définies à partir des caractéristiques du site et des critères économiques de l'époque, peuvent ètre plus ou moins temporairement modifiées en fonction de l'hydraulicité du réseau hydrographique ou de la disponibilité des autres sources de production d'électricité.

Dans la mesure où la marge de manouvre de l'exploitant est très réduite, la limitation de l'impact entraîné par la régulation du débit de la rivière passe obligatoirement par un choix de l'objectif prioritaire d'utilisation de l'eau : exutoire pour les déchets domestiques ou source de production d'énergie électrique.

La compatibilité de ces 2 usages avec la sauvegarde du cours d'eau est possible moyennant des mesures souvent très onéreuses comme :

- l'épuration des eaux résiduaires pour limiter les charges polluantes,

- la restitution au barrage d'un débit réservé suffisant pour diluer la pollution.

\section{Bibliographie}

BARANOV I.V., 1961. - The zoning of the USSR in to hydrochemical zones and provinces in terms of the nutrient content and photosynthetic activity of plankton inhabiting their waters. - In : Pervichanaya produktsia morey $i$ vnutrennykhvodoyemov : 97-101, Minsk.

BENSON N.G., 1973a. - Man-made lakes as aquatic ecosystems In : W.C. Ackermann, G.F. White and E.B. Worthington eds, Man made lakes: their problems and environmental effects : 683-689. Amer Geophys. Union, Washington, D.C.

BENSON N.G., 1973b. - Evaluating the effects of discharges rates, water levels, and peaking on fish populations in Missouri River Main Stem Impoundments. In : W.C. Ackermann, G.F. White and E.B. Worthington eds., Man made lakes, Their problems and environmental effects : 683-689 Amer Geophys. Union. Washington. D.C.

BENZECRI J.P., 1972. - Sur l'analyse des tableaux binaires associés à une correspondance multiple. Note multigraphiée du Laboratoire de Statistique mathématique. Univ. Paris VI.

CAILLEUX A., 1954. - Limites dimensionnelles et noms des fractions granulométriques. Bull. Soc. Géol. Fr., 4 : 643646.
CHAMPEAU A., GREGOIRE A. et BRUN G., 1979. - Le peuplement piscicole des lacs artificiels du Verdon. Annls Limnol 14 (3) : 245-271.

CHAMPEAU A. et al., 1980. - Les retenues hydro-électriques du Verdon. Impact sur la rivière - Conséquences du marnage. Rapport Com. Faune-Flore Minis. Environ. : 98 p.

CHERRY D.S. et GUTHRIE R.K., 1975. - Significance of detritus or detritus associated invertebrates to fish production in a new impoundment. J. Fish Res. Bd. Can., 32: 1799-1804.

CLAVEL P. et BOUCHAUD B., 1980. - Incidences des extractions de granulats d'alluvions et de certains travaux hydrauliques, sur le périphyton, la production primaire et la production secondaire dans trois cours d'eau du Massif Central. Annls Stat. Limnol. Besse-en-Chandesse, 14:211-350.

COSTE M., 1974. - Etude sur la mise au point d'une méthode biologique de détermination de la qualité des eaux en milieu fluvial; DQEPP, CTGREF, Paris : $78 \mathrm{p}$.

DIDAY E., 1970. - La méthode des nuées dynamiques. Revue de Statistique appliquée, 19 (2).

DUSSART B., 1966. - Limnologie - L'étude des eaux continentales. Gauthier - Villars éd., Paris : $667 \mathrm{p}$.

EDMONDS J.S. et WARD J.V., 1979. - Profundal benthos of a multibasin foothills reservoir in Colorado, USA Hydrobiologia, 63 (3) : 199-208.

FEUILLADE J, et FEUILLADE M., 1971. - Etude comparative en 1966 et 1967, de trois lacs de retenues du projet de parc naturel régional du Morvan. Annls Hydrobiol., 2 (2) : 143174.

FISHER S.G. et LAVOY A., 1972. - Differences in littoral fauna due to fluctuating water levels below a hydroelectric dam. J. Fish. Res. Bd. Can., 29 : 1472-1476.

GAGNEUR J., 1976. - Etude des diptères du Lot et étude de la retenue de Cajarc. Thèse $3^{\mathrm{e}}$ cycle, Toulouse $: 189 \mathrm{p}$.

GORE J.A., 1977. - Reservoir manipulations and benthic macroinvertebrates in a prairie reservoir. Hydrobiologia, 55 (2) : 113-123.

GORE J.A., 1980. - Ordinational analyses of benthic communities upstream and downstream of a prairie storage reservoir. Hydrobiologia, 69 (1-2) : 33-44.

GREGOIRE A., 1975. - Effets de la vidange décennale de la retenue de la Chaudanne sur les biocénoses du Verdon (octobre 1974). Rev. Biol. Ecol. Medit., 2 (2) : 15-26.

GREGOIRE A. et CHAMPEAU A., 1981. - Les Ephéméroptères d'une rivière à débit régulé : le Verdon. Verh. Internat. Verein. Limnol., $21: 857-865$.

GREGOIRE A., RIVIER B. et RONDON J., 1975. - Etude écologique d'une petite retenue : le seuil de Gréoux, Rev. BiolEcol. Medit., 2 (3) : 19-34.

HOLDEN P.B., 1979. - Ecology of riverine fishes in regulated stream systems. In : J.V. Ward and J.A. Stanford eds., The ecology of regulated streams : 57-74. Plenum Press, NewYork.

HUTCHINSON G.E. et LOFFLER H., 1956. - The thermal stratification of lakes. Proc. nat. Acad. Sci., Wash., 42 : 84-86.

Figure 6 - Impact des barrages sur le cours d'eau aval. Analyse factorielle des correspondances multiples. Position des stations d'étude* et des modalités correspondant à l'ensemble des composantes de l'écosystème Verdon sur le plan 1-3.

$\mathrm{DEBI}=$ débit, $\mathrm{VITE}=$ vitesse, $\mathrm{COND}=$ conductivité, $\mathrm{CL}=$ chlore, $\mathrm{NA}=$ sodium, $\mathrm{K}=$ potassium, $\mathrm{SO}_{4}=$ sulfates, $\mathrm{PO} 4=$ phosphates, $\mathrm{NO}_{3}=$ nitrates, $\mathrm{CAMG}=$ dureté, $\mathrm{HCO}_{3}=$ alcalinité, $\mathrm{PH}=\mathrm{pH}, \mathrm{O} 2 \mathrm{DI}=$ oxygène, $\mathrm{TEMP}=$ température, $\mathrm{SIO}_{2}=$ silice, $\mathrm{ALAN}=A c h-$ nanthes lanceolata, $\mathrm{AMIN}=A$. minutissima, $\mathrm{AOVA}=$ Amphora ovalis, $\mathrm{APEL}=$ Amphipleura pellucida, $\mathrm{AFOL}=$ Anomoconeis follis, $\mathrm{CARC}=$ Ceratoneis arcus, $\mathrm{CPED}=$ Cocconeis pediculus, $\mathrm{CKUT}=$ Cyclotella kützingiana, $\mathrm{CAFF}=$ Cymbella affinis, $\mathrm{CPRO}=$ C. prostata, $\mathrm{CDEL}=C$. delicatula, $\mathrm{CSIN}=C$. sinuata, $\mathrm{DTIS}=$ Denticula tenuis, $\mathrm{DVUL}=$ Diatoma vulgare, $\mathrm{DTEN}=D$. tenue, $\mathrm{DOVA}=$ Diplo neis ovalis, FPIN $=$ Fragilaria pinnata, GOLI $=$ Gomphonema olivaceum, NGRA $=$ Navicula gracilis, NCRY $=N$. cryptocephala, $\mathrm{NAMP}=$ Nitzschia amphibia, $\mathrm{NDIS}=N$. dissipata, $\mathrm{NROM}=N$. romana, $\mathrm{RCUR}=$ Rhoicosphenia curvata, $\mathrm{SACU}=$ Synedra accus, $\mathrm{SRUMP}=S$. rumpens, SOVA $=$ Surirella ovata, $\mathrm{CMOE}=$ Caenis moesta, $\mathrm{EIGN}=$ Ephemerella ignita, ORHE = Oligoneuriella rhenana, PLUT $=$ Potamanthus luteus, $\mathrm{BLUT}=$ Baetis lutheri, $\mathrm{BMEL}=B$. melanony $x, \mathrm{BALP}=B$. alpinus, $\mathrm{BSIN}=B$. sinaicus, $\mathrm{BMUT}=$ Baetis muticus, BVER = Baetis vernus, ETOR = Epeorus torrentium, RAUR = Rhithrogena aurantiaca, PSUB = Paraleptophlebia submarginata, $\mathrm{EFOR}=$ Ecdyonurus forcipula, $\mathrm{AROB}=$ Acanthocyclops robustus, $\mathrm{MALB}=$ Macrocyclops albidus, $\mathrm{BAFF}=$ Biapertura affinis, OSTRA = Ostracodes, GPUL $=$ Gammarus pulex, DYTI $=$ Dy tiscidés, ODON = Odonates, HIRU $=$ Hirudinés, DRYO = Dryopidés, PLEC = Plécoptères, AANG = Anguilla anguilla, BBAR = Barbus barbus, TSOU = Leuciscus soufia, LCEP =L. cephalus, CGOB = Cottus gobio, $\mathrm{GGOB}=$ Gobio gobio, $\mathrm{CNAS}=$ Chondrostoma nasus, $\mathrm{CTOX}=C$. toxostoma, $\mathrm{SBIP}=$ Spirlinus bipunctatus, NBAR $=$ Cobitis barbatula, $\mathrm{PPHO}=$ Phoxinus phoxinus, $\mathrm{ZASP}=$ Zingel asper . 
HYNES H.B.N., 1961. - The effect of water-level fluctuations on littoral fauna. Verh. Internat. Verein. Limnol., $14: 652$ 656.

KASTER J.L. et JACOBI G.Z., 1978. - Benthic macroinvertebrates of a fluctuating reservoir. Freshwat. Biol., $8: 283-290$.

LAIR N., 1975. - Rôle du zooplancton dans les réseaux trophiques de deux lacs du Massif Central français. Thèse Doct. Clermont Ferrand : $166 \mathrm{p}$.

LAIR N. et al. 1980. - Etude hydrobiologique de la retenue de Chastang (Dordogne). Doc. EDF. Non publié.

LAMBERTI E. et NINO A., 1979. - Etude du lac de SerrePonçon : domaine benthique. Rev. Biol. Ecol. Medit., (3-4): 256.

LAURENT M. et MOREAU G., 1973. - Influence des facteurs écologiques sur le coefficient de condition d'un téléostéen (Cottus gobio L.). Annls Hydrobiol., 4 (2) : 211-228.

LEMKUHL D.M., 1972. - Changes in thermal regime as a cause of reduction of benthic fauna downstream of a reservoir. J. Fish. Res. Bd. Can,, $29: 1329-1332$.

LOKHANINA L.A., 1959. - Preliminary data of the formation of zooplankton in the Gor'Ky Reservoir. Trudy VI Sovershchaniya po probl Biolog. vnutrennigh vod : $340-346$

LOUCKS O.L. et ODUM W.E., 1978. - Analysis of five North American lake ecosystems. I - A strategy for comparison. Verh. Internat. Verein. Limnol., 20:556-561.

LOWE-Mc CONNELL R.H., 1973. - Reservoirs in relation to man fisheries. - In : W.C. Ackermann, G.F. White and E.B Worthington eds., Man made lakes : Their problems and environmental effects : 641-654. Amer. Geophys. Union, Washington. D.C.

LUND J.W.G., 1978. - Changes in the phytoplankton of an English lake, 1945-1977. Hydrobiol. J., 14 (1): 6-21.

MACAN T.T., 1974. - Freshwater ecology. Longman Group. ed., London : $343 \mathrm{p}$.

MARGALEF R., 1975 - Typology of reservoirs. Verh. Internat. Verein. Limnol., $19: 1841-1848$.

MARSHALL B.E., 1978. - Aspects of the ecology of benthic fauna in lake Mc Ilwaine. Rhodesia. Freswat. Biol., 8 : 241-249.

MILLERIOUX G., GREGOIRE A. et CHAMPEAU A., 1981. Les populations de Diatomées d'une rivière à débit régulé : Le Verdon. Annls Limnol., 17 (1) : 63-77.

MINSHALL G.W. et WINGER P.V., 1968. - The effect of reduction in stream flow on invertebrate drift. Ecology. 49 580-582.

NAUMANN E, 1932. - Grundzuge der regional en Limnologie Binnengew. $11(9): 176$ p.

NINO A., 1979. - Approche d'une étude de la faune benthique des lacs de barrage. Influence des marnages sur les conditions de vie et de survie des larves de chironomides. DEA., Marseille, Univ. Provence : $53 \mathrm{p}$

NISBET M., 1961. - Un exemple de pollution de rivière pat vidange d'une retenue hydroélectrique. Verh. Internat. Verein. Theor. Angew. Limnol, $14: 678-680$.

PARSONS T.R. et STRICKLAND J.D.H., 1963. - Discussion of spectrophotometric determination of marine-plant pigments with revised equations for ascertaining chlorophylls and carotenoids. J. Mar. Res., 21 (3) : 155-163.

PETR T., 1978. - Tropical man-made lakes - Their ecological impact. Arch. Hydrobiol., 81 (3) : 368-385.

RADFORD D.S. et HARTLAND-ROWE R, 1971. - A preliminary investigation of bottom fauna and invertebrate drift in an unregulated and a regulated stream in Alberta. $J . A p p l$. Ecol., 8 (3) : 883-903.
RYALS G.L., 1975. - Density and distribution of benthos in lake Keowee, South Carolina. Ph. D. Dissertation. Clemson Univ., Clemson, South Carolina : 86 p.

SAKAMOTO M., 1966. - Primary production by phytoplankton community in some Japanese Lakes and its dependence on Lake depth. Arch. Hydrobiol., $62: 1-28$.

SLADECEK V., 1963. - Schema biologiokeho trideni vod. Vod. Hospod., $11: 421-422$.

SPENCE J.A. et HYNES H.B.N., 1971. - Differences in benthos upstream and downstream of an impoundment. J. Fish. Res. Bd. Can., $28: 35-43$.

SPILLMAN C.J., 1961. - Poissons d'eau douce. Faune de France, 65 303 p.

STEEMANN NIELSEN E., 1952. - The use of radio-active carbon $\left({ }^{14} \mathrm{C}\right)$ for measuring organic production in the sea. J. Cons. Explor. Mer. 18:117-140.

STRASKRABA M., 1978. - Theoretical considerations on eutrophication. Verh. Internat. Verein. Limnol, $20: 2714$ 2720.

THIENEMANN A., 1925. - Die Binnengewäser Mitteleuropas, eine limnologische Einfühning, Binnengew., $1: 255$ p.

THUT R.N., 1969. - A study of the profundal bottom fauna of lake Washington. Ecol. Monogr., 39 : 79-100.

UTERMOHL H., 1958. - Zur Vervollkommung der quantitative Phytoplankton Methodik, Mitt. Intern. Verein. Limnol., 9 : $1-38$.

UZABIAGA G., 1978. - Morphométrie, physiconchimie et phy toplancton de trois lacs de barrage d'altitude des Pyrénées. Thèse $3^{\mathrm{e}}$ cycle, Toulouse : $111 \mathrm{p}$.

VERNEAUX J , 1973 - Cours d'eau de Franche-Comté Massif du Jura), Recherches écologiques sur le réseau hyđrographique du Doubs - essai de biotypologie. Thèse, Annls Scient Univ. Besançon, Zool., Physiol., Biol. Anim., 3 (9) : 260 p.

VIVIER P. et al., 1956. - Le lac d'Eguzon - Annls Stat. Cent. Hydrobiol. Appl., $6: 1-230$.

WALKER J.H. et LAWSON J.D., 1977. - Natural stream temperature variations in a catchment. Wat. Res., $14: 373-377$.

WARD J.V, 1974. - A temperature-stressed stream ecosystem below a hypolimnial release mountain reservoir. Arch. Hydrobiol. 74 (2) : 247-275.

WARD J.V., 1976a. - Comparative limnology of differentially regulated sections of a Colorado mountain river. Arch. Hydrobiol, 78 (3) : 319-342.

WARD J.V., 1976b. - Effects of thermal constancy and seasonal temperature displacement on community structure of stream macroinvertebrates. - In : G.W. Esch and R.W. Mc Farlane eds., Thermal ecology II : 302-307. ERDA Symposium series 40 .

WARD J.V. et SHORT R.A., 1978. - Macroinvertebrate community structure of four special lotic habitats in Colorado, U.S.A. Verh. Internat. Verein. Limnol., 20:1382-1387.

WARD J.V. et STANFORD J.A., 1979. - Ecological factors controlling stream zoobenthos. - In : J.V. Ward et J.A. Stanford eds., The ecology of regulated streams : 35-55. Plenum Press, New-York.

WEBSTER J.R., BENFIELD E.F. et CAIRNS J., 1979. - Model predictions of the effects of stream regulation on particulate organic matter transport. In : J.V. Ward and J.A. Stanford eds., The ecology of regulated streams : 339-364. Plenum Press, New-York.

WHIPPLE G.C., 1927. - The microscopy of drinking water. J. Wiley ed., New-York : 586 p. 